\title{
Effect of Organic and Potassium Fertilization on Productivity and Quality of Sugar Beet in Sandy Soil
}

\author{
${ }^{*}$ Yussef, H. I, ${ }^{\star}$ F. I. Radwan, ${ }^{\star *}$ M. A. Gomaa and ${ }^{\star *}$ M. M. Abdel- Rahman \\ * Nubaria Agriculture Research Station. Institute of sugar crops. \\ ** Plant Production Department. Faculty of Agriculture (Saba- Basha). Univ. Alexandria
}

\begin{abstract}
Two field experiments were carried out at the Experimental Farm of ElNubaria Agriculture Research station, Alexandria, Egypt, at the Kilometer 71 North west to study the effect of potassium fertilizers and organic manure (Farmyard manure) on yield and quality of sugar beet (var. Kumara) during the winter seasons of 2014/2015 and 2015/2016. The experimental design was split plot design with three replicates. The main results could be summarized as followers: (1) All characters for yield and quality was significantly affected by potassium fertilization. Application of $60 \mathrm{~kg} \mathrm{~K} 2 \mathrm{O} / \mathrm{fed}$, gave the greatest values of root length, top yield/fed, root yield/fed biological yield/fed and sugar yield/fed as well as sucrose $\%$ and TSS\% in the both seasons except purity\% in the two seasons. (2) All characters increased by increasing rate of organic manure up to $10 \mathrm{~m}^{3} / \mathrm{fed}$, except purity $\%$ in the two seasons. (3) The interaction indicated that the highest all yield sucrose $\%$ and TSS\% was obtained by application $60 \mathrm{~kg} \mathrm{~K} \mathrm{~K}_{2} \mathrm{O} / \mathrm{fed}$, with rate of $10 \mathrm{~m}^{3} / \mathrm{fed}$ farmyard manure in both seasons. The farmyard manure plays a major role in crop production in deserts soils sence it inirriazant the use of chemical fertilizer and decreases environmental pollution.
\end{abstract}

Key words: Sugar beet, Potassium levels, Organic manure, yields Quality.

\section{INTRODUCTION}

Sugar beet (Beta vulgaris, L.) is one of the two crops (the older being sugar cane) which represent the important source of sucrose product. The importance of sugar beet crop to agriculture is not only confined to sugar production, but also it well known to be adapted to poor, saline, alkaline and calcareous soil.

The economic maybe increasing sugar productivity could be achieved through development appropriate new technology package for sugar beet crop that includes agronomic management to the yield and quality of sugar beet (Mokadem, 1993, Kandil et al., 2002 and Esmail and Abo El- Hamd, 2007).

Potassium plays a fundamental role in sucrose synthesis and storage. The influence of potassium not only on carbohydrate assimilation but also in nitrogen metabolism (Abdel Rahiman, 1996, El- Maghraby et al., 1998) mentioned that plant length, root diameter, root, top and sugar yield/fed, as well as sucrose and T.S.S. percentage significantly increased by increasing potassium level up to $48 \mathrm{~kg} \mathrm{~K} \mathrm{~K}_{2} \mathrm{O} / \mathrm{fed}$. On the other hand, Hegazy et al. (1992), found that there was significant decrease in top and sugar yields by increasing potassium level from 0 up $45 \mathrm{~kg} \mathrm{~K} \mathrm{O} /$ fed and added that sucrose and purity percentage were not significantly affected by potassium rates.

The organic manure is known by enhancing soil physical properties by increasing the moisture holding capacity. In addition, it can change the chemical properties of soil through lowering $\mathrm{pH}$ and extensively their beneficial effects are known for long time. Application of organic matter provides many essential nutrients needed by plants. The increase in crop yield due to using of animal 
manure have been imperative many times as resulted manily from the nitrogen, phosphorus or potassium on the combination of the three mentioned elements (Negm et al., 2003). Zalat and Nemeat Allah (2001) reported that farmyard manure (FYM) increased sucrose\% and T.S.S\%.

Therefore, the investigation was designed to study the effect of potassium fertilization and organic manure on yield and quality of sugar beet crop.

\section{MATERIALS AND METHODS}

Two filed experiments were carried out through two successive season of 2014/2015 and 2015/2016 at the Experimental farm Station Research, ElNubaria, Buhyra, Egypt at the $71^{\text {th }} \mathrm{Km}$ West Alexandria- Cairo deresat road. To investigate the effect of potassium fertilizer and organic manure levels and their interaction of yield and quality on sugar beet (Beta vulgaris, L.) var. kumara.

Maize (Zea mays, L.) was the preceding for the two seasons. The experimental design was split plot design with three replicates. Potassium fertilization (zero, 20, 40 and $60 \mathrm{~kg} \mathrm{~K} \mathrm{O} / \mathrm{fed}$ ) occupied the main plots. The subplot were assigned to three organic manure (sheep catle manure) (Untreated, 5 and $10 \mathrm{~m}^{3} / \mathrm{fed}$ ). Some physical and chemical properties of the experimental field soil and organic matter (farm yard manure) during the two seasons were done and the data are shown in Tables (1 and 2).

Potassium sulfate $\left(48 \% \mathrm{~K}_{2} \mathrm{O}\right)$ was applied at how many rates. Nitrogen fertilizer was added in the form of ammonium nitrate $(33.5 \% \mathrm{~N})$ as a side dressing at the rate of $60 \mathrm{~kg} \mathrm{~N} / \mathrm{fed}$, in two equal parts, one after thinning (before the first irrigation and the other before the second irrigation. Calcium super phosphate $\left(15.5 \% \mathrm{P}_{2} \mathrm{O}_{5}\right)$, was applied during tillage operation at the rate of 100 $\mathrm{kg} / \mathrm{fed}$. Seeds ball were hand sown at the usual dry sowing on one side of the redge in hills $25 \mathrm{~cm}$ apart at the rate of $4-5$ seed ball per hill on $3^{\text {rd }}$ and $14^{\text {th }}$ September in 2014/2015 and 2015/2016 seasons, respectively. The experimental basic unit area was $10.5 \mathrm{~m}^{2}$ (1/400 feddan) and includes 6 redges each of which $50 \mathrm{~cm}$ width and 3 meter length.

At harvest (200 days after sowing) five plants were chosen at random from the iner redges of each sub- plot to estimate yield components and quality characters as follows: 
Table (1). some physical and chemical properties of the experimental soil in 2014/2015 and 2015/2016 seasons

\begin{tabular}{|c|c|c|}
\hline \multirow{2}{*}{\multicolumn{3}{|c|}{$\begin{array}{l}\text { Soil properties } \\
\text { A- Mechanical analysis }\end{array}$}} \\
\hline & & \\
\hline Sand\% & 85.70 & 88.23 \\
\hline Clay\% & 6.30 & 4.80 \\
\hline Silt\% & 8.00 & 6.97 \\
\hline Soil texture & sandy & sandy \\
\hline \multicolumn{3}{|l|}{ B- Chemical analysis } \\
\hline $\mathrm{pH}(1: 1)$ & 8.50 & 7.35 \\
\hline $\mathrm{EC}(\mathrm{dS} / \mathrm{m})$ & 1.20 & 1.14 \\
\hline \multicolumn{3}{|l|}{ 1- Soluble cations (1:2) (cmol/kg soil) } \\
\hline $\mathrm{K}^{+}$ & 0.82 & 1.20 \\
\hline $\mathrm{Ca}^{++}$ & 2.76 & 3.10 \\
\hline $\mathrm{Mg}^{++}$ & 1.90 & 2.30 \\
\hline $\mathrm{Na}^{++}$ & 4.35 & 4.65 \\
\hline \multicolumn{3}{|l|}{ 2- Soluble anions $(1: 2)(\mathrm{cmol} / \mathrm{kq}$ soil) } \\
\hline $\mathrm{CO}_{3}^{-}+\mathrm{HCO}_{3}^{-}$ & 2.72 & 2.72 \\
\hline $\mathrm{CL}^{-}$ & 7.90 & 7.09 \\
\hline $\mathrm{SO}_{4}^{-}$ & 1.15 & 0.98 \\
\hline Calcium carbonate (\%) & 6.12 & 6.72 \\
\hline Total nitrogen $(\mathrm{mg} / \mathrm{kg})$ & 33.00 & 23.00 \\
\hline Available Phosphorus (mg/kg) & 3.17 & 3.14 \\
\hline Organic matter (\%) & 0.37 & 0.83 \\
\hline
\end{tabular}

Table (2). Some chemical properties of farmyard manure

\begin{tabular}{lcc}
\hline & Analysis & Values \\
\hline Moisture \% & 27.00 \\
$\mathrm{O} . \mathrm{M} . \%$ & 26.00 \\
$\mathrm{pH}(1: 1)$ & 7.20 \\
$\mathrm{~N} \%$ & 2.06 \\
$\mathrm{P} \%$ & 3.13 \\
$\mathrm{~K} \%$ & 1.48 \\
$\mathrm{C}: \mathrm{N}$ raito & $7.32: 1$ \\
\hline
\end{tabular}

1- Top yield (ton/fed).

2- Root yield (ton/fed)

3- Biological yield (ton/fed).

4- Sugar yield (ton/fed).

5- Sucrose\%: it was determing according to Mc Ginnu (1971).

6- Juice purity\%: It was calculated according to Le - Decte (1927)

$$
\text { Juice purity } \%=\frac{\text { Sucrose } \%}{\text { T.S.S. } \%} \times 100
$$

7- Total soluble solids (T.S.S.\%)

$$
=\frac{\text { Sucrose } \%}{\text { Purity } \%}
$$




\section{Statistical analysis:}

All collected data here subjected to the statistical and analysis following the procedure described by Gomez and Gomez (1984). The least significantly differences test (L.S.D.) at 0.05 was used to compare between means of the different treatments.

\section{RESULTS AND DISCUSSION}

\section{A- Effect of potassium fertilization on yield and quality:}

Data presented in Tables ( 3 and 4 ) revealed that all character of yield and quality were significantly affected by potassium levels in both seasons. A gradual increase to root length, top yield/fed, root yield/fed, biological yield, sugar yield/fed, sucrose $\%$, purity\% and T.S.S.\% increased as K- levels raised from 0 to $60 \mathrm{~kg} \mathrm{~K} 2 \mathrm{O} / \mathrm{fed}$, in the both seasons. Such increase in root yield/fed, mounted by $28.13,35.50$ and $60.83 \%$ in the first season, being 20.56, 32.29 and $67.15 \%$ in the second season, as K- levels raised from 0 to 20 and $60 \mathrm{~kg}$ $\mathrm{K}_{2} \mathrm{O} / \mathrm{fed}$. Similar significant increase in sugar yield/fed, amounted to 45.83 , $48.74 \%$ and $80 \%$ in the first season, being $29.24,37.28$ and $78.39 \%$ compared to control in the second season. These results could be attributed to the important role of potassium in physiological process in plant such as translocation of sugar and carbohydrates of assimilates from the top to the root (Ibrahim et al., 2002). Also, its role in nutritional balance, which increased organic compounds through phytosynthesis (El- Howary, 1999). Similar results were obtained by Mekki and El- Gazzar (1999), Omar et al. (2002) and Esmail and Abo El- Hamd (2007).

Data presented in Tables (3 and 4) showed that, root length, top yield/fed, root yield/fed, biological yield, sugar yield/fed, sucrose\% purity\% and T.S.S.\% were affected significantly by tested organic manure during the two growing seasons. Application of $10 \mathrm{~m}^{3} / \mathrm{fed}$, organic manure gave the tallest roots $(32.75$ and $35.33 \mathrm{~cm}$ ) heaviest top yield/fed $(9.70$ and 7.75 ton), heaviest root yield (26.96 and 24.46 ton), heaviest biological yield/fed (35.8 and 32.26 ton), highest sugar yield (4.85 and 4.35 ton), highest sucrose\% (18.25 and $17.59 \%)$ purity\% (86.22 and $84.90 \%)$ and highest T.S.S.\% (21.17 and $21.50 \%)$ in the first and second seasons, it could be concludes that treated of traits with organic fertilizer levels on increase yield and quality characters. This may be due to the role of microorganisms activity, phytohormones formation and translocation of the plant especially (IAA, Gas and CKs). Also, it has important role in increasing photosynthesis rate. These results are similar to those of Bassal et al (2001), Ali (2003) and Ibrahim (2007).

The interaction between potassium levels and organic manure levels had significant effect on all yield and quality character except purity\% in both seasons. Application of $60 \mathrm{~kg} \mathrm{~K} \mathrm{~K}_{2} \mathrm{O} / \mathrm{fed}$, gave the highest values for this traits except purity \% treated with $10 \mathrm{~m}^{3} / \mathrm{fed}$, resulted the maximum mean in both seasons Tables (4 and 6). Finally it could be concluded that under condition of this study the highest root and sugar yield/fed produced by application of $60 \mathrm{~kg}$ $\mathrm{K}_{2} \mathrm{O} / \mathrm{fed}$ treated with $10 \mathrm{~m}^{3} / \mathrm{fed}$ 
Table (3). Yield and its components as affected by potassium fertilizer and organic manure in 2014/2015 and 2015/2016 seasons

\begin{tabular}{|c|c|c|c|c|c|c|c|c|c|c|}
\hline \multirow[t]{2}{*}{ Treatments } & \multicolumn{2}{|c|}{$\begin{array}{l}\text { Root length } \\
\text { (cm) }\end{array}$} & \multicolumn{2}{|c|}{$\begin{array}{l}\text { Top yield } \\
\text { (ton/fed) }\end{array}$} & \multicolumn{2}{|c|}{$\begin{array}{c}\text { Root yield } \\
\text { (ton/fed) }\end{array}$} & \multicolumn{2}{|c|}{$\begin{array}{c}\text { Biological yield } \\
\text { (ton/fed) }\end{array}$} & \multicolumn{2}{|c|}{$\begin{array}{c}\text { Sugar yield } \\
\text { (ton/fed) }\end{array}$} \\
\hline & $2014 / 2015$ & $2015 / 2016$ & $2014 / 2015$ & $2015 / 2016$ & $2014 / 2015$ & $2015 / 2016$ & $2014 / 2015$ & $2015 / 2016$ & $2014 / 2015$ & $2015 / 2016$ \\
\hline \multicolumn{11}{|l|}{ A) K- fertilizer } \\
\hline 0 & $25.56^{d}$ & $28.45^{\mathrm{C}}$ & $6.14^{c}$ & $5.36^{d}$ & $14.68^{c}$ & $14.40^{\mathrm{d}}$ & $20.92^{d}$ & $19.71^{d}$ & $2.40^{\mathrm{C}}$ & $2.36^{\mathrm{c}}$ \\
\hline 20 & $30.22^{c}$ & $31.11^{\mathrm{b}}$ & $7.83^{b}$ & $5.91^{\mathrm{c}}$ & $18.81^{\mathrm{b}}$ & $17.36^{\mathrm{c}}$ & $26.61^{\mathrm{c}}$ & $23.25^{\mathrm{c}}$ & $3.50^{\mathrm{b}}$ & $3.05^{\mathrm{b}}$ \\
\hline 40 & $32.33^{b}$ & $32.00^{\mathrm{b}}$ & $8.25^{b}$ & $6.67^{b}$ & $19.82^{b}$ & $19.05^{b}$ & $28.13^{\mathrm{b}}$ & $25.81^{\mathrm{b}}$ & $3.57^{b}$ & $3.24^{b}$ \\
\hline 60 & $34.49^{a}$ & $35.00^{a}$ & $9.41^{\mathrm{a}}$ & $7.98^{\mathrm{a}}$ & $23.61^{a}$ & $24.07^{a}$ & $32.78^{a}$ & $32.05^{a}$ & $4.32^{\mathrm{a}}$ & $4.21^{\mathrm{a}}$ \\
\hline LO.S.D. (0.05) & 1.50 & 1.60 & 0.50 & 0.54 & 1.20 & 1.50 & 1.39 & 2.05 & 0.50 & 0.55 \\
\hline \multicolumn{11}{|l|}{ B) Organic manure } \\
\hline Control & $26.42^{c}$ & $27.25^{\mathrm{c}}$ & $7.12^{b}$ & $5.60^{b}$ & $12.31^{c}$ & $12.53^{c}$ & $19.40^{c}$ & $18.03^{c}$ & $2.02^{c}$ & $22.03^{c}$ \\
\hline $5 \mathrm{~m}^{3} / \mathrm{fed}$ & $30.75^{\mathrm{b}}$ & $32.34^{b}$ & $6.91^{\mathrm{b}}$ & $6.12^{b}$ & $18.43^{b}$ & $19.18^{b}$ & $25.13^{b}$ & $25.26^{\mathrm{b}}$ & $3.24^{b}$ & $3.23^{\mathrm{b}}$ \\
\hline $10 \mathrm{~m}^{3} / \mathrm{fed}$ & $33.75^{\mathrm{a}}$ & $35.33^{a}$ & $9.70^{\mathrm{a}}$ & $7.72^{\mathrm{a}}$ & $26.96^{\mathrm{a}}$ & $24.46^{a}$ & $36.80^{a}$ & $32.26^{a}$ & $4.95^{\mathrm{a}}$ & $4.39^{a}$ \\
\hline L.S.D. (0.05) & 2.20* & $2.40^{*}$ & $0.72^{*}$ & $0.65^{*}$ & $2.40^{*}$ & 2.70* & $4.80^{*}$ & $4.40^{*}$ & $0.80^{*}$ & $0.65^{*}$ \\
\hline \multicolumn{11}{|l|}{ Interations } \\
\hline AxB & * & * & * & * & * & * & * & * & * & * \\
\hline
\end{tabular}

Means of each designated by the same letter not significantly different at $5 \%$ using least significant difference L.S.D.

* Significant at 0.05 levels of probability 
Table (4). Interaction between potassium fertilizer and organic manure in 2014/2015 and 2015/2016 seasons on yield and components

\begin{tabular}{|c|c|c|c|c|c|c|c|c|c|c|c|}
\hline \multicolumn{2}{|c|}{ Treatments } & \multicolumn{2}{|c|}{$\begin{array}{l}\text { Root length } \\
\text { (cm) }\end{array}$} & \multicolumn{2}{|c|}{$\begin{array}{l}\text { Top yield } \\
\text { (ton/fed) }\end{array}$} & \multicolumn{2}{|c|}{$\begin{array}{c}\text { Root yield } \\
\text { (ton/fed) }\end{array}$} & \multicolumn{2}{|c|}{$\begin{array}{c}\text { Biological yield } \\
\text { (ton/fed) }\end{array}$} & \multicolumn{2}{|c|}{$\begin{array}{c}\text { Sugar yield } \\
\text { (ton/fed) }\end{array}$} \\
\hline $\begin{array}{c}\text { Org. } \\
\text { manure }\end{array}$ & $\begin{array}{c}\text { K- } \\
\text { levels }\end{array}$ & $2014 / 2015$ & $2015 / 2016$ & $2014 / 2015$ & $2015 / 2016$ & $2014 / 2015$ & $2015 / 2016$ & $2014 / 2015$ & $2015 / 2016$ & $2014 / 2015$ & $2015 / 2016$ \\
\hline \multirow{4}{*}{ Control } & control & 25.00 & 25.00 & 5.73 & 5.38 & 10.28 & 9.14 & 16.01 & 14.18 & 1.51 & 1.43 \\
\hline & 20 & 25.00 & 25.67 & 7.40 & 5.33 & 10.76 & 10.76 & 18.10 & 16.10 & 1.72 & 1.76 \\
\hline & 40 & 28.33 & 28.33 & 7.63 & 4.70 & 12.58 & 13.52 & 20.53 & 18.20 & 2.13 & 2.23 \\
\hline & 60 & 29.33 & 30.00 & 7.70 & 6.98 & 15.64 & 16.64 & 22.95 & 23.62 & 2.17 & 2.71 \\
\hline \multirow{4}{*}{$5 \mathrm{~m}^{3} / \mathrm{fed}$} & control & 25.00 & 26.67 & 5.76 & 5.43 & 12.30 & 13.95 & 18.04 & 19.38 & 2.01 & 2.23 \\
\hline & 20 & 31.00 & 32.67 & 6.84 & 6.18 & 17.82 & 17.82 & 24.67 & 23.86 & 3.21 & 3.09 \\
\hline & 40 & 33.33 & 33.33 & 6.44 & 6.44 & 18.18 & 18.86 & 34.61 & 25.30 & 3.21 & 3.14 \\
\hline & 60 & 33.67 & 36.67 & 8.09 & 6.43 & 25.08 & 26.09 & 33.21 & 32.51 & 4.53 & 4.44 \\
\hline \multirow{4}{*}{$10 \mathrm{~m}^{3} / \mathrm{fed}$} & control & 26.67 & 33.67 & 6.93 & 5.26 & 21.12 & 20.12 & 28.11 & 25.38 & 3.67 & 3.41 \\
\hline & 20 & 34.67 & 35.00 & 9.24 & 6.23 & 27.85 & 23.51 & 37.05 & 29.74 & 4.98 & 4.29 \\
\hline & 40 & 35.33 & 34.33 & 10.19 & 8.86 & 28.72 & 24.72 & 39.25 & 33.92 & 5.36 & 4.36 \\
\hline & 60 & 40.33 & 38.33 & 12.45 & 10.54 & 30.47 & 29.47 & 42.18 & 40.01 & 5.71 & 5.48 \\
\hline L.S.D & 0.05 & $2.30^{\star}$ & $2.50^{\star}$ & $0.80^{\star}$ & $0.85^{\star}$ & $2.50^{\star}$ & $2.75^{\star}$ & $3.70^{\star}$ & $4.20^{\star}$ & $0.95^{\star}$ & $0.70^{\star}$ \\
\hline
\end{tabular}


Table (5). Sugar beet quality as affect by potassium fertilizer and organic manure in 2014/2015 and 2015/2016 seasons

\begin{tabular}{|c|c|c|c|c|c|c|}
\hline \multirow{2}{*}{ Treatments } & \multicolumn{2}{|c|}{ Sucrose \% } & \multicolumn{2}{|c|}{ Purity \% } & \multicolumn{2}{|c|}{ T.S.S. \% } \\
\hline & $2014 / 2015$ & $2015 / 2016$ & $2014 / 2015$ & $2015 / 2016$ & $2014 / 2015$ & $2015 / 2016$ \\
\hline \multicolumn{7}{|l|}{ A) K- fertilizer } \\
\hline 0 & $16.00^{c}$ & $16.45^{c}$ & $81.12^{\mathrm{d}}$ & $80.03^{d}$ & $19.89^{b}$ & $19.89^{c}$ \\
\hline 20 & $17.33^{\mathrm{b}}$ & $16.89^{b}$ & $83.20^{\mathrm{b}}$ & $81.20^{\mathrm{C}}$ & $20.78^{\mathrm{a}}$ & $21.00^{\mathrm{a}}$ \\
\hline 40 & $17.78^{\mathrm{b}}$ & $17.22^{c}$ & $82.70^{\mathrm{C}}$ & $81.70^{\mathrm{b}}$ & $20.55^{\mathrm{a}}$ & $20.55^{\mathrm{b}}$ \\
\hline 60 & $18.11^{\mathrm{a}}$ & $17.33^{\mathrm{a}}$ & $83.80^{a}$ & $82.40^{\mathrm{a}}$ & 20.86 & $21.33^{\mathrm{a}}$ \\
\hline LO.S.D. (0.05) & 0.38 & 0.35 & 0.40 & 0.42 & 0.50 & 0.45 \\
\hline \multicolumn{7}{|l|}{ B) Organicmanure } \\
\hline Control & $16.25^{\mathrm{c}}$ & $16.25^{\mathrm{c}}$ & $84.20^{\mathrm{b}}$ & $82.60^{\mathrm{C}}$ & $20.09^{b}$ & $20.20^{c}$ \\
\hline $5 \mathrm{~m}^{3} / \mathrm{fed}$ & $17.42^{\mathrm{b}}$ & $17.08^{\mathrm{b}}$ & $85.16^{\mathrm{ab}}$ & $83.40^{\mathrm{b}}$ & $20.08^{\mathrm{b}}$ & $20.58^{\mathrm{b}}$ \\
\hline $10 \mathrm{~m}^{3} / \mathrm{fed}$ & $18.25^{\mathrm{a}}$ & $17.59^{\mathrm{a}}$ & $86.22^{\mathrm{a}}$ & $84.90^{a}$ & $21.17^{\mathrm{a}}$ & $21.50^{\mathrm{a}}$ \\
\hline L.S.D. (0.05) & $0.60^{*}$ & $0.45^{\star}$ & $0.65^{\star}$ & $0.50^{\star}$ & $0.60^{*}$ & $0.52^{\star}$ \\
\hline \multicolumn{7}{|l|}{ Interations } \\
\hline AxB & * & * & ns & ns & * & * \\
\hline
\end{tabular}

Table (6). Interaction between potassium fertilizer and organic manure on quality of sugar beet in 2014/2015 and 2015/2016 seasons

\begin{tabular}{|c|c|c|c|c|c|}
\hline \multicolumn{2}{|c|}{ Treatments } & \multicolumn{2}{|c|}{ Sucrose \% } & \multicolumn{2}{|c|}{$\begin{array}{c}\text { Total soluble soild } \\
\text { (T.S.S.\%) }\end{array}$} \\
\hline Org. manure & K- levels & $2014 / 2015$ & $2015 / 2016$ & 2014/2015 & $2015 / 2016$ \\
\hline \multirow{4}{*}{ Control } & control & 14.67 & 15.67 & 20.00 & 19.33 \\
\hline & 20 & 16.00 & 16.33 & 20.67 & 20.33 \\
\hline & 40 & 17.00 & 16.67 & 20.00 & 20.00 \\
\hline & 60 & 17.33 & 16.33 & 19.67 & 20.33 \\
\hline \multirow{4}{*}{$5 \mathrm{~m}^{3} / \mathrm{fed}$} & control & 16.00 & 16.67 & 19.33 & 20.00 \\
\hline & 20 & 18.00 & 17.33 & 20.33 & 21.00 \\
\hline & 40 & 17.67 & 17.33 & 20.33 & 20.33 \\
\hline & 60 & 18.00 & 17.00 & 20.33 & 21.00 \\
\hline \multirow{4}{*}{$10 \mathrm{~m}^{3} / \mathrm{fed}$} & control & 17.33 & 17.00 & 20.33 & 20.33 \\
\hline & 20 & 18.00 & 17.67 & 21.33 & 21.67 \\
\hline & 40 & 18.67 & 17.67 & 21.00 & 21.33 \\
\hline & 60 & 19.00 & 18.67 & 22.00 & 22.67 \\
\hline \multicolumn{2}{|c|}{ L.S.D. 0.05} & $0.70^{*}$ & $0.50^{\star}$ & $0.70^{\star}$ & $0.60^{*}$ \\
\hline
\end{tabular}

\section{REFERENCES}

Abdel- Rahman, M. M. (1996). The effect of N, P and K fertilization on growth, yield and some physiological characters of sugar beet (Bela vulgaris, L.), M. SC. Thesis, Fac. Of Agric. Zagazig Univ., Egypt.

Ali, M. H. A. (2003). Microbiological chemical studies on the rhizophere of sugar beet plant. Ph. D. Thesis, Fad. Agric. Al- Azhar Univ.

Bassal, S. A. A., A. A. Zohry and K. A. Deuby (2001). Effect of row and hill spacing and biomineral $\mathrm{N}$ fertilization rates on sugar beet productivity $\mathrm{J}$. Agric. Sci. Mansoura Univ., 26 (9): 5217- 5226. 
El- Hawary, M. A. (1999). Influence of nitrogen, potassium and boron fertilizer levels on sugar beet under salire soil condition J. Agric. SCl. Mansoura Univ., 24 (4): 1573- 1581.

El- Maghraby, S. S., M. M. Shehata and Y. H. Tawfik (1998). Effect of soil and foliar application of nitrogen and potassium on sugar beet. Egypt. J. Agric. Res., 75 (2): 665- 679.

Esmail, A. A. and A. S. Abo El- Hamd (2007). Effect of sowing dates, hill spacing and potassium fertilization on yield, yield components and quality of sugar beet (Beta vulgaris, L.) undere El- Minia Governorate conditions, J. Agric. SCl. Mansura, Univ. 32 (3): 1627- 1638.

Gomez, K. A. and A. A. Gomez (1984). Statistical Produces for agricultural Research An Interation al. Rce. Research Institute Book John Wiley and Sons Inc. New York.

Hegazy, M. H., M. S. M. Abo-Sliman, K. M. Sayed and M. A. Abo El- Soud (1992). Effect of rate and time of K- fertilization on yield and quality of sugar beet. Egypt. J. Appl. Sci., 7 (4): 396- 403.

Ibrahim, M. M., M. R. Khalifa, M. A. Koriem F. I. Zein and E. H. Omar (2002). Yield and quality of sugar beet crop as affected by mid to late season drought and potassium fertilization a North Nile Delta Egyptian J. of soil Sci., 42.(1): 87- 102.

Ibrahim, O. M. (2007). Effect of potassium and organic fertilizer on the productivity of some sugar beet varieties under saline conditions ph. D. Thesis, Fac. Of Agric. Alex. Univ., Egypt.

Kandil, M. S., E. A. Mohamoud; A. A. El- Gharbawy and A. A. Hassanein (2002). Response of sugar beet to levels and times of potassium fertilization under salinity condition at North Delta of Egypt. J. Agric. Sci., Mansoura Univ., 27 (1): 7237- 7240.

Le- Docte, A. (1927). Commercial determination of sugar beet root using the sech Le- Docte process. Sugar J., 29: 488- 492.

Mc- Ginnus, R. A. (1971). Sugar beet technology $2^{\text {nd }}$ and sugar beet development found, fort colins. Color. USA.

Mekki, B. B. and M. M. El- Gazzar (1999). Response of root yield quality of sugar beet (Beta vulgaris, L.) to irrigation with saline water ahd foliar potassium fertilization. Ann. of Agric. Sci., 44 (1): 213- 225.

Mokadem, Sh. A. (1993). Evaluation of some sugar beet vareties with varying plant Po. Under El- Minia Governo rate condition. Assiut. J. Agric. Sci., 24 (4): $77-93$.

Omar, E. H., M. A. Ghazy, M. A. A. Abd Allah and M. M. Regab (2002). Response of sugar to termination of last irrigation, hill spacing and Kfertilization J. Agric. Sci. Mansoura Univ., 27 (6): 4291 - 4303. 
الملخص العربي

تأثير التسميد العضوي والبوتاسي على الإنتاجية وجودة بنجر السكر في

الأرض الرملية

\section{"حسن يوسف إبراهيم يوسف "*"تحي إبراهيم رضوان "**ممود عبد العزيز جمعة "محمد مصطفى عبد الرحمن}

* مركز بحوث بالنوبارية - معهد المحاصيل السكرية

** قسم الإنتاج النباتي - كلية الزراعة سابا باشا - جامعة الإسكندرية - مصر برد

أجريت تجربتان حقليتان بالمزرعة البحثية بمركز البحوث الزراعية بالنوبارية - البحيرة - عند الكيلو

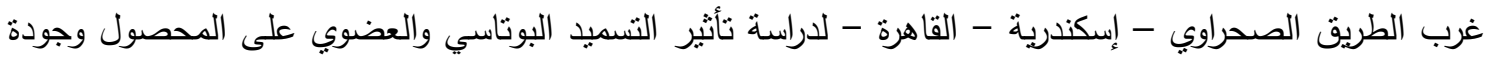

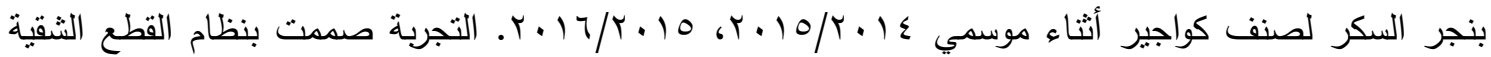
مرة واحدة مع ثلاث مكررات.

ويمكن تلخيص أهم النتائج فيما يلي: مدرة

- جميع الصفات للمحصول والجودة كانت متأثرة معنوياً بواسطة التسميد البوتاسي عند إضافة ـ اكجم بو بأ/ فدان أعطت أفضل قيم لطول الجذر محصول العروش/فدان، محصول الجذور/فدان والمحصول البيولوجي/فدان، محصول السكر/فدان وأيضا النسبة المئوية للسكر والنسبة المئوية للمواد الصلبة في كلا الموسمين ماعدا النسبة لجنة المئوية للنقاوة في الموسمين. - جميع الصفات زادت بواسطة زيادة السماد العضوي عند ـامَ/فدان ماعدا النسبة المئوية للنقاوة في كلا الموسمين. - أدى التداخل إلى ارتفاع كل صفات المحصول والنسبة المئوية للسكروز والنسبة المئوية للمواد الصلبة الذائبة

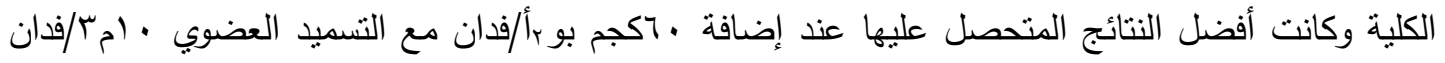
(سماد بلدي) في كلا الموسمين. - السماد البلدي تحت الدراسة له تأثثر كبير في زيادة إنتاج المحصول خصوصاً في الأرضي الصحراوية نظراً

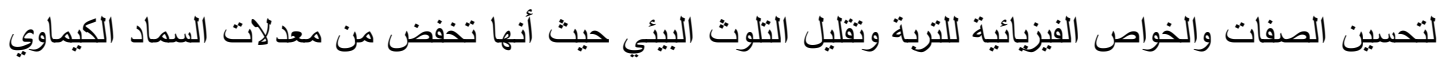
(طريقة تسميد صديقة للبيئة). 
